\title{
AVALIAÇÃO DE CRITÉRIOS DE HETEROGENEIDADE BASEADOS EM ATRIBUTOS MORFOLÓGICOS PARA SEGMENTAÇÃO DE IMAGENS POR CRESCIMENTO DE REGIÕES
}

Evaluation of Heterogeneity Criteria Based on Morphological Attributes for Image Segmentation by Region Growing

\author{
RODRIGO DA SILVA FERREIRA ${ }^{1}$ \\ GILSON A. O. P. COSTA ${ }^{1}$ \\ RAUL QUEIROZ FEITOSA ${ }^{1,2}$ \\ ${ }^{1}$ Pontifícia Universidade Católica do Rio de Janeiro - PUC-Rio \\ Rio de Janeiro - RJ - Brasil \\ ${ }^{2}$ Universidade Estadual do Rio de Janeiro - UERJ \\ Rio de Janeiro - RJ - Brasil \\ (rsilva, gilson,raul)@ele.puc-rio.br
}

\begin{abstract}
RESUMO
Avalia-se neste trabalho o impacto de se considerar atributos morfológicos na formulação do critério que governa o crescimento de regiões na segmentação de imagens. Para tanto, uma extensão do algoritmo de segmentação multiresolução proposto por Baatz e Schäpe (2000) foi proposta e implementada, permitindo que se testassem critérios derivados de diferentes atributos morfológicos. O estudo valeu-se de um método supervisionado para medir numericamente a qualidade da segmentação. $O$ resultado ideal da segmentação foi representado por um conjunto de segmentos de referência delineados manualmente para três recortes de imagens Quickbird-2. Para cada critério testado, os valores ótimos para os parâmetros do algoritmo de segmentação foram determinados por um processo estocástico que procurou minimizar a discrepância entre as referências e o resultado de cada segmentação. Uma análise tanto quantitativa quanto qualitativa dos resultados indicou inequivocamente que a inclusão de atributos morfológicos no critério de heterogeneidade, que decide a fusão entre segmentos adjacentes no processo de crescimento de regiões, pode resultar numa substancial melhoria da qualidade da segmentação. $\mathrm{O}$ artigo realça ainda a importância de se adotar atributos
\end{abstract}


morfológicos apropriados para cada classe de objetos e tece considerações que orientam a escolha destes atributos.

Palavras-chave: Segmentação de Imagens; Avaliação da Qualidade da Segmentação; Análise de Imagens Baseada em Objetos Geográficos; Extração de Atributos.

\section{ABSTRACT}

This study assesses the impact of using morphological attributes in the formulation of the criterion that rules the region growing process in image segmentation. Therefore, an extension to the multi-resolution segmentation algorithm proposed by Baatz and Schäpe (2000) was proposed and implemented, allowing testing criteria derived from different morphological attributes. The study used a supervised method to measure the segmentation quality numerically. The ideal segmentation result was represented by a set of reference segments delineated manually for three Quickbird-2 image subsets. For each tested criterion, the optimal values of the segmentation algorithm parameters were determined by a stochastic process that aimed at minimizing the discrepancy between the references and the result of each segmentation. Quantitative and qualitative analysis of the results indicated unequivocally that the introduction of morphological attributes in the heterogeneity criterion, which drives the merging of adjacent segments in the region growing process, can result in a substantial improvement in segmentation quality. The paper highlights the importance of adopting morphological attributes suitable for each object class and discusses the selection of such attributes.

Keywords: Image Segmentation; Segmentation Quality Assessment; Geographic Object-Based Image Analysis; Feature Extraction.

\section{INTRODUÇÃO}

Desde o lançamento do satélite IKONOS em 1999, tem crescido a disponibilidade de imagens da superfície da Terra geradas por sensores orbitais de altíssima resolução espacial. Se por um lado tal aumento na oferta de imagens passou a viabilizar um grande número de novas aplicações, antes restringidas pelos altos custos das imagens aéreas, por outro lado tornou evidente as limitações das técnicas de análise de imagens baseadas na radiometria do pixel (BLASCHKE; STROBL, 2001). Esta percepção foi a principal motivação para a emergência da área de investigação científica que passou a chamar-se GEOBIA ou OBIA (BLASCHKE; LANG; HAY, 2008), e que tem como principal característica a classificação de aglomerados de pixels que formam segmentos, ao invés da classificação de pixels isolados. Um dos pressupostos básicos desta abordagem é que segmentos têm maior conteúdo semântico do que pixels individuais, o que se expressa, por exemplo, em atributos morfológicos.

Ao longo das últimas décadas vários algoritmos de segmentação foram propostos (HARALICK; SHAPIRO, 1985; TILTON, 1989; BAATZ; SCHÄPE, 
2000; COMANICIU; MEER, 2002; VESE; CHAN, 2002; FELZENSZWALB; HUTTENLOCHER, 2004; MEINEL; NEUBERT, 2004; NEUBERT; HEROLD; MEINEL, 2006; FORCADEL; GUYADER; GOUT, 2008; NEUBERT; HEROLD; MEINEL, 2008; MARPU et al., 2010). Um estudo conduzido por Neubert, Herold e Meinel (2006) indicou o algoritmo implementado no software SPRING (CÂMARA et al., 1996) e o algoritmo proposto por Baatz e Schäpe (2000), disponível no software eCognition, como os dois melhores algoritmos de segmentação dentre os mais usados nos anos recentes na área de sensoriamento remoto. Ambos os algoritmos têm em comum o paradigma de "crescimento de regiões". Diferem, no entanto, quanto ao critério que decide quando dois segmentos adjacentes devem ou não ser mesclados formando um único segmento. Enquanto o algoritmo do SPRING considera basicamente apenas atributos espectrais, o algoritmo de Baatz e Schäpe considera tanto atributos espectrais quanto morfológicos. Este diferencial confere, em princípio, ao algoritmo de Baatz e Schäpe maior capacidade de produzir resultados condizentes com a percepção visual humana, mas torna, por outro lado, o ajuste de seus parâmetros mais complexo e laborioso.

Tal algoritmo tem sido aplicado em diversos problemas nos campos de sensoriamento remoto (ALVES et al., 2009) e análise de imagens médicas (BAUM; KULKARNI; CARRERAS, 2012). Apesar do interesse alcançado por este segmentador na comunidade científica, não se tem notícia de estudos que comprovem o benefício para a qualidade da segmentação de considerarem-se atributos morfológicos além de atributos espectrais para medir a heterogeneidade de segmentos.

Outra questão em aberto diz respeito à escolha dos atributos morfológicos. $\mathrm{O}$ algoritmo de segmentação em questão considera apenas a suavidade e a compacidade. Os autores do presente trabalho desconhecem estudos que justifiquem tal opção, e questionam se outros atributos morfológicos poderão ser mais adequados do que estes, dependendo da classe de objetos a ser reconhecida.

O presente estudo aborda tais questões e tem como objetivo avaliar o impacto relativo da utilização de diferentes atributos morfológicos no processo de segmentação. Propõe-se neste trabalho uma extensão ao algoritmo de Baatz e Schäpe que comporta uma maior gama de atributos morfológicos para descrição de heterogeneidade. A extensão foi implementada em software e diversas combinações de atributos morfológicos foram testadas num conjunto de três imagens de sensoriamento remoto contendo alvos com formas distintas.

O restante deste documento está organizado da seguinte forma. O texto começa com a apresentação do método de segmentação proposto e dos atributos morfológicos utilizados neste trabalho. Depois, é mostrada a função de avaliação da qualidade da segmentação que será utilizada e o método de otimização escolhido para selecionar os valores dos parâmetros de segmentação. A seção seguinte apresenta os experimentos e os resultados obtidos. $\mathrm{Na}$ última seção, são apresentadas as conclusões e apontados possíveis desdobramentos deste trabalho. 


\section{ALGORITMO DE SEGMENTAÇÃO MULTIRESOLUÇÃO ESTENDIDO}

Nesta seção, são apresentados o método de segmentação de imagens proposto neste trabalho e os atributos morfológicos utilizados.

\subsection{Processo de Segmentação}

$\mathrm{O}$ algoritmo de segmentação de imagens proposto neste trabalho é uma extensão do algoritmo de crescimento de regiões proposto por Baatz e Schäpe (2000).

No início do processo de segmentação cada pixel da imagem constitui uma semente e representa um segmento. Segue-se à inicialização um processo iterativo. A cada iteração, cada segmento é visitado uma única vez seguindo uma ordem pseudoaleatória, de modo a favorecer um crescimento equilibrado de todos os segmentos e a reprodutibilidade dos resultados.

Ao se visitar um segmento, calcula-se o aumento de heterogeneidade que resultaria de sua fusão com cada um de seus vizinhos. Determina-se então o vizinho mais similar, ou seja, aquele cuja fusão implicaria no menor acréscimo de heterogeneidade em relação a cada segmento considerado separadamente. Adota-se em seguida uma entre duas heurísticas de decisão. Na variante conhecida como Melhor Ajuste (Best Fitting), realiza-se a fusão do segmento visitado com seu vizinho mais similar. Na segunda variante, conhecida como Melhor Ajuste Mútuo (Mutual Fitting), a fusão somente se consuma, se a relação de maior similaridade for mútua, ou seja, se o segmento visitado for da mesma forma o vizinho mais similar de seu vizinho mais similar. Ressalte-se que para ambas as heurísticas, a fusão está ainda condicionada a que o correspondente acréscimo da heterogeneidade seja inferior ao quadrado de um parâmetro do algoritmo chamado escala $(E)$ que determina o máximo aumento de heterogeneidade permitido, resultante da fusão entre dois segmentos, e que influencia indiretamente no tamanho médio dos segmentos finais (BRODSKÝ; BORU゚VKA, 2006). A segmentação termina quando mais nenhuma fusão puder ser realizada.

Como mostra a Equação 1, a medida do acréscimo de heterogeneidade $f$, também chamada fator de fusão, possui uma componente espectral $h_{c o r}$ e uma morfológica $h_{\text {forma }}$. A importância relativa de cada componente é dada pelo peso da forma $w_{\text {forma }}$, outro parâmetro do algoritmo. Assim, o fator de fusão é dado por:

$$
f=\left(1-w_{\text {forma }}\right) \cdot h_{\text {cor }}+w_{\text {forma }} \cdot h_{\text {forma }} .
$$

A componente espectral do fator de fusão é definida por uma combinação linear dos valores de desvio-padrão das intensidades dos pixels que compõem o segmento em cada banda. Os coeficientes da combinação linear são parâmetros definidos pelo usuário, que expressam a contribuição relativa de cada banda espectral da imagem para a heterogeneidade do segmento. A formulação da componente espectral é dada pela Equação 2, onde $\mathrm{Seg}_{1}$ e $\mathrm{Seg}_{2}$ referem-se aos 
segmentos considerados para fusão, e $\mathrm{Seg}_{3}$ ao segmento resultante da fusão de $\mathrm{Seg}_{1}$ com $\mathrm{Seg}_{2}$, formalmente:

$$
h_{c o r}=\sum_{c} w_{c}\left(n_{\text {Seg } 3} \cdot \sigma_{c}^{\operatorname{Seg} 3}-\left(n_{\operatorname{Seg} 1} \cdot \sigma_{c}^{\text {Seg } 1}+n_{\text {Seg } 2} \cdot \sigma_{c}^{\text {Seg } 2}\right)\right)
$$

Nessa equação, $c$ e $w_{c}$ denotam, respectivamente, a banda espectral e o peso a ela associado, $\sigma_{c}$ é o desvio-padrão dos valores dos pixels que compõem cada segmento na banda $c$, e $n_{S e g}$ é o número de pixels do segmento. No algoritmo os pesos $w_{c}$ das bandas são não negativos e normalizados de modo a somarem 1 (um). Admite-se, ao mesmo tempo, que os pesos $w_{c}$ estão expressos em unidades tais que tornam $h_{c o r}$ adimensional.

A componente morfológica é definida pelo desvio relativo da forma do segmento em relação a formas geométricas pré-definidas. $\mathrm{O}$ algoritmo original considera dois atributos morfológicos: compacidade e suavidade. A extensão introduzida neste trabalho aumenta o conjunto de atributos morfológicos que podem ser considerados para o cálculo da componente morfológica. A seção seguinte apresentará as formulações dos dois atributos usados no algoritmo original e dos novos atributos sugeridos na extensão aqui proposta.

A formulação da componente morfológica $h_{\text {forma }}$ é generalizada de modo que, não apenas dois, mas vários atributos morfológicos podem ser utilizados, como mostra a Equação 3:

$$
h_{\text {forma }}=\sum_{s} w_{s}\left(n_{\text {Seg } 3} \cdot a_{s}^{\text {Seg } 3}-\left(n_{\text {Seg } 1} \cdot a_{s}^{\text {Seg } 1}+n_{\text {Seg } 2} \cdot a_{s}^{\text {Seg } 2}\right)\right),
$$

Nessa equação, $s$ é um índice que indica um dos atributos morfológicos, $a_{s}$ é o valor do $s$-ésimo atributo, e $w_{s}$ o peso não negativo a ele associado, sendo que $\sum_{s} w_{s}=1$.

Tanto a componente $h_{\text {cor }}$ quanto $h_{\text {forma }}$ são medidas do acréscimo da heterogeneidade resultante da fusão de dois segmentos - heterogeneidades associadas à cor e à forma, respectivamente. Essas componentes são ponderadas pela área em pixels dos segmentos, de forma que cada segmento tenha a mesma importância relativa, contribuindo de forma equivalente para a heterogeneidade média global da segmentação resultante (BAATZ; SCHÄPE, 2000).

\subsection{Atributos Morfológicos}

As seções a seguir apresentam inicialmente a definição dos dois atributos que têm sido utilizados para compor a heterogeneidade morfológica. Em seguida apresentam-se as definições de mais sete atributos de forma acrescidos neste estudo ao algoritmo de segmentação. Cabe notar, em primeiro lugar, que todos os atributos são adimensionais. Em segundo lugar, deve-se ressaltar que todos os atributos a seguir são definidos de modo a alcançarem seu valor mínimo teórico quando a 
forma do segmento coincide perfeitamente com uma forma de referência (p.ex. círculo, retângulo, etc.). Em vista disso, a definição de alguns dos atributos apresentados nesta seção pode diferir da definição que se encontra em outras publicações da área.

\subsubsection{Compacidade}

Representa o desvio em relação a um quadrado (BAATZ; SCHÄPE, 2000). É dada pela razão entre o comprimento $l$ da borda do segmento e a raiz quadrada da sua área, esta dada pelo número $n$ de pixels contidos no segmento, ou seja:

$$
a_{\text {compacidade }}=\frac{l}{\sqrt{n}}
$$

A compacidade é mínima para um quadrado e é sensível a irregularidades de contorno.

\subsubsection{Suavidade}

Mede o quanto o objeto se afasta de sua envoltória convexa. É dada pela razão entre o comprimento $l$ da borda do segmento e o comprimento $l_{\text {rem }}$ da borda do retângulo envolvente mínimo, formalmente:

$$
a_{\text {suavidade }}=\frac{l}{l_{\text {rem }}}
$$

A suavidade é mínima para um retângulo perfeito e cresce com a presença de concavidades. Deve-se ressaltar aqui a diferença em relação à definição apresentada em (BAATZ; SCHÄPE, 2000) para suavidade. Esta se baseia no chamado retângulo envolvente, cujos eixos são paralelos aos lados da imagem, enquanto que o termo retângulo envolvente mínimo se refere ao retângulo envolvente cujos lados são paralelos aos eixos da elipse que apresenta momento de segunda ordem idêntico ao do segmento.

\subsubsection{Retangularidade}

Corresponde à dissimilaridade em relação a uma forma retangular (HALCON, 2012. Adaptado). É dada pela razão entre a área do retângulo envolvente mínimo $n_{\text {rem }}$ e a área do segmento, como mostra a Equação 6:

$$
a_{\text {retangularidade }}=\frac{n_{\text {rem }}}{n}
$$

A retangularidade é igual a 1 (um) para um retângulo perfeito e aumenta com a presença de reentrâncias. Ė sensível à presença de ramificações finas e longas. 


\subsubsection{Isometria}

Representa o desvio em relação a uma forma isométrica (HALCON, 2012). É dada pela razão entre o semieixo principal $a$ e secundário $b$ da elipse com mesmo momento de segunda ordem, isto é:

$$
a_{\text {isometria }}=\frac{a}{b}
$$

A isometria é igual a 1 (um) para um objeto isométrico e aumenta à medida que ele se alonga.

\subsubsection{Anisometria}

É o inverso da isometria, portanto:

$$
a_{\text {anisometria }}=\frac{b}{a}
$$

A anisometria é igual a 1 (um) para um objeto isométrico e diminui à medida que ele se alonga.

\subsubsection{Bulkiness}

Representa o desvio em relação a um círculo com raio de comprimento intermediário entre os semieixos principal $a$ e secundário $b$ da elipse com mesmo momento de segunda ordem (HALCON, 2012), conforme mostra a Equação 9:

$$
a_{\text {bulkiness }}=\frac{\pi \cdot a \cdot b}{n}
$$

\subsubsection{Excentricidade}

Trata-se de mais um parâmetro que visa capturar o quanto a forma do objeto difere da de um círculo, como mostra a Equação 10:

$$
a_{\text {excentricidade }}=\sqrt{1-\left(\frac{b}{a}\right)^{2}}
$$

A excentricidade é igual a 0 (zero) para um círculo perfeito e tende a 1 (um) quando $\mathrm{o}$ alongamento tende a infinito.

\subsubsection{Roundness}

Novamente neste caso, mede-se o desvio em relação a um círculo, em particular com diâmetro igual ao eixo principal $a$ da elipse com mesmo momento de segunda ordem (RUSS, 1998). A Equação 11 define formalmente este atributo.

Bol. Ciênc. Geod., sec. Artigos, Curitiba, v. 19, no 3, p.452-471, jul-set, 2013. 


$$
a_{\text {roundness }}=\frac{\pi \cdot(2 a)^{2}}{4 \cdot n}
$$

Roundness é igual a 1 (um) para um círculo perfeito e aumenta à medida que o eixo principal aumenta em relação à área. Desta forma, é mais sensível ao alongamento do objeto (GOMES; PACIORNIK, 2005).

\subsubsection{Fator de Forma Circular}

Como o nome sugere, este atributo também mede o desvio em relação a um círculo (RUSS, 1998) a partir da seguinte formulação matemática:

$$
a_{\text {fator_circular }}=\frac{l^{2}}{4 \cdot \pi \cdot n}
$$

É mínimo para um círculo perfeito e aumenta à medida que o comprimento $l$ da borda do segmento aumenta em relação à sua área. Dessa maneira, o fator de forma circular é mais sensível a irregularidades de contorno (GOMES; PACIORNIK, 2005).

\section{AVALIAÇÃO DA QUALIDADE DA SEGMENTAÇÃO}

Nesta seção, são apresentados a métrica de discrepância utilizada para a avaliação da qualidade da segmentação e o método de otimização escolhido para o ajuste automático dos parâmetros de segmentação.

\subsection{Métrica de Discrepância}

No presente trabalho, pressupõe-se a existência de um conjunto de polígonos de referência que representam o resultado ideal de uma segmentação. Diferentemente de abordagens não supervisionadas (CHABRIER et al., 2006), no método aqui adotado a qualidade da segmentação é dada por uma medida de discrepância quanto à forma entre cada polígono de referência e o segmento que a ele corresponde. Diversas medidas de discrepância foram propostas na literatura para tal propósito (BEAUCHEMIN; THOMPSON, 1997; CLINTON et al., 2010; LIU et al., 2012). Em estudos anteriores (FERREIRA, 2011), os autores compararam diversas métricas e concluíram que a medida de discrepância proposta em (FEITOSA et al., 2006) apresenta entre todas as alternativas avaliadas a maior correlação com a percepção do olho humano. Essa métrica, denominada Reference Bounded Segments Booster (RBSB), foi adotada no presente trabalho e é descrita sucintamente a seguir.

Seja $r_{i}$ o $i$-ésimo polígono de um conjunto $R$ de referências e $s_{k}$ o $k$-ésimo elemento do conjunto $S$ dos segmentos produzidos por um dado segmentador aplicado a uma imagem de entrada. Com algum abuso de notação, mas sem prejuízo 
da clareza, $r_{i}$ e $s_{k}$ denotam ao mesmo tempo o conjunto de pixels contidos respectivamente em $r_{i}$ e $s_{k}$. O segmento correspondente à referência $r_{i}$ é o segmento que possui a maior interseção com referência $r_{i}$, formalmente:

$$
k_{i}=\underset{k}{\operatorname{argmax}}\left\{\#\left(r_{i} \cap s_{k}\right)\right\}
$$

$\mathrm{Na}$ equação, $s_{k} \in S$ e \#(·) representa o operador cardinalidade. Assim, o índice de discrepância $D$ utilizado neste trabalho é dado por:

$$
D=\frac{1}{\#(R)} \sum_{r_{i} \in R} \frac{\#\left[\left(r_{i} \cup s_{k_{i}}\right)-\left(r_{i} \cap s_{k_{i}}\right)\right]}{\#\left(r_{i}\right)}
$$

Claramente, o valor mínimo de $D$ é 0 (zero) e ocorre quando o resultado da segmentação se ajusta perfeitamente às referências.

\subsection{Ajuste Automático dos Parâmetros de Segmentação}

O método de otimização usado para encontrar automaticamente os valores dos parâmetros de segmentação foi a Busca de Padrões Generalizada (do inglês Generalized Pattern Search - GPS). Este pertence a um subconjunto dos métodos de busca direta chamado busca de padrões (do inglês pattern search) e foi proposto por Torczon (1997) como uma generalização de métodos anteriores (HOOK; JEEVES, 1961) e do algoritmo de busca multidirecional (DENNIS; TORCZON, 1991).

Os algoritmos GPS calculam uma sequência de soluções que se aproximam do ótimo global iterativamente. A cada iteração, o algoritmo seleciona uma malha de soluções ao redor da solução corrente. Se uma solução melhor do que a solução corrente é encontrada, aquela se torna a nova solução corrente para a próxima iteração. Caso contrário, a solução corrente permanece como tal para a iteração seguinte.

Para selecionar soluções putativas a serem avaliadas a cada iteração, o algoritmo se desloca da solução corrente no espaço de busca em diversas direções definidas por um conjunto de vetores, cuja magnitude é dada por um fator chamado tamanho da malha. Quando se encontra uma solução melhor numa iteração, o tamanho da malha aumenta, caso contrário, diminui. $\mathrm{O}$ algoritmo termina quando o tamanho da malha alcança um limiar mínimo definido a priori.

\section{AVALIAÇÃO EXPERIMENTAL}

Nesta seção são avaliados os resultados obtidos com o algoritmo de segmentação de imagens proposto neste trabalho. Os resultados são analisados quantitativamente e em alguns casos qualitativamente a partir de uma avaliação visual. 
Na seção 4.1 é apresentado o banco de imagens utilizado nos experimentos. As seções subsequentes descrevem o procedimento experimental, os resultados e as conclusões deles derivadas. Duas séries de experimentos foram realizadas, envolvendo a seleção dos valores ótimos para os parâmetros de segmentação. Na primeira série de experimentos (seção 4.2) foram realizadas segmentações que consideraram apenas atributos espectrais e segmentações em que, além destes, um atributo morfológico foi introduzido no critério de heterogeneidade. Na segunda série de experimentos (seção 4.3), foram considerados dois atributos morfológicos em conjunto com os atributos espectrais.

\subsection{Banco de Imagens}

Os experimentos para avaliação do método proposto foram realizados em um conjunto de três recortes de imagens Quickbird-2, cujas bandas RGB foram fusionadas com a banda pancromática, produzindo uma resolução espacial de $0,61 \mathrm{~m}$. Para cada recorte, segmentos de referência foram delineados manualmente por um analista. As classes de objetos-alvo selecionadas para o experimento foram: tanques, telhados e árvores (Figura 1).

Figura 1 - Imagens de teste: tanques (a), telhados (b) e árvores (c), e respectivas referências.
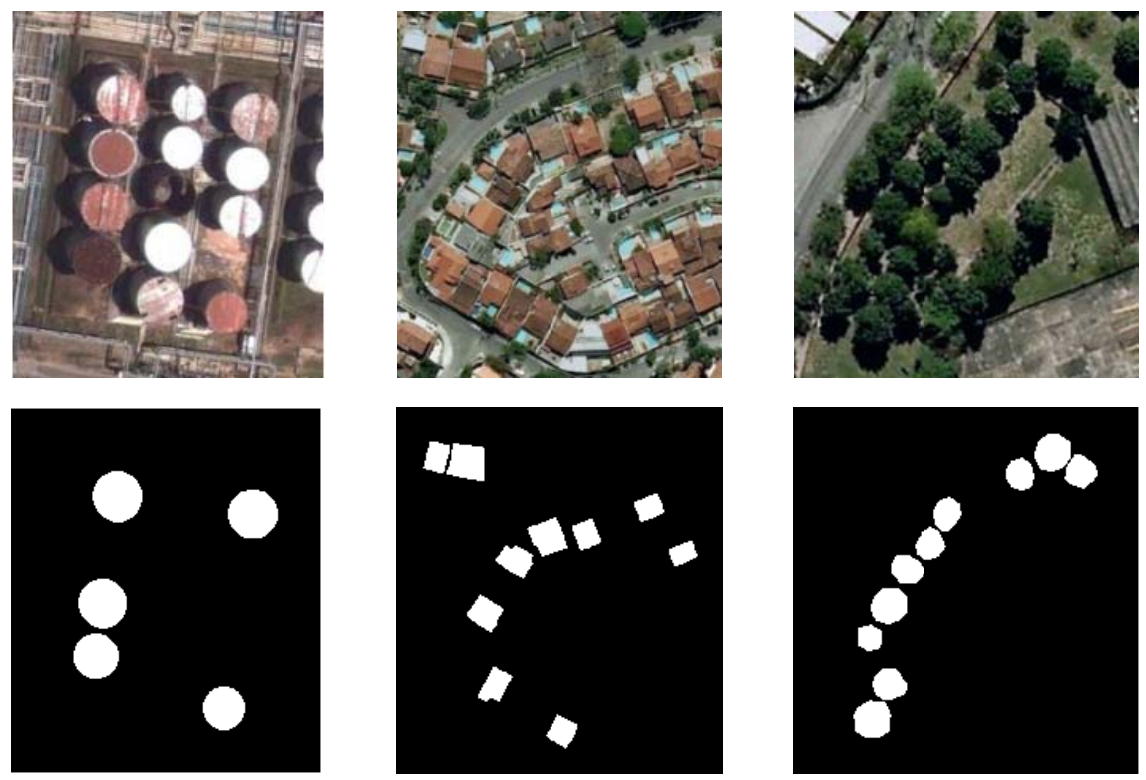

(a)

(b)

(c) 
A Figura 1 apresenta as três imagens utilizadas e as respectivas referências. A imagem (a) é um recorte de 219x257 pixels, de um conjunto de tanques da refinaria do município de Duque de Caxias - RJ. Delinearam-se cinco referências de tanques. A imagem (b) é um recorte de 350×393 pixels, de uma área residencial na Barra da Tijuca, na cidade do Rio de Janeiro. Foram delineadas 10 referências de telhados cerâmicos. A terceira imagem (c) é um recorte de 272×287 pixels, de uma área residencial da Barra da Tijuca, também na cidade do Rio de Janeiro. Nesta imagem, delinearam-se dez referências de árvores isoladas. Apenas as bandas RGB foram consideradas nos experimentos.

\subsection{Segmentação com base em um atributo morfológico e em atributos espectrais}

Inicialmente, avaliaram-se segmentações realizadas usando apenas atributos espectrais. O valor ótimo do parâmetro de escala $E$ foi estimado pelo método GPS. $\mathrm{O}$ peso da forma $w_{\text {forma }}$ foi fixado em 0 (zero), de modo a excluírem-se atributos morfológicos da medida de heterogeneidade, e adotou-se um mesmo valor para os pesos de todas bandas espectrais. Tendo em vista que o método de otimização utilizado é estocástico, para cada imagem, tomou-se o melhor resultado de dez experimentos.

Em seguida, um único atributo morfológico foi acrescido aos atributos espectrais já utilizados nos experimentos anteriores para definir o critério de heterogeneidade nas segmentações. Desta forma, dois foram os parâmetros estimados: o parâmetro de escala $E$ e o peso forma $w_{\text {forma }}$. Novamente, os pesos das bandas assumiram todos um mesmo valor e o peso $w_{s}$ foi fixado em 1 (um), de modo a se considerar apenas um atributo morfológico. $\mathrm{O}$ objetivo desta etapa foi verificar, no primeiro momento, se a introdução de um atributo morfológico levaria a segmentações de melhor qualidade (menor discrepância) do que aquelas realizadas considerando-se apenas a cor. Num segundo momento, verificou-se qual entre os atributos morfológicos descritos na seção 2 mais contribuiria para a qualidade da segmentação para cada imagem.

Novamente neste caso, para cada imagem de teste e para cada atributo morfológico, adotou-se como resultado a menor discrepância alcançada em dez experimentos executados. Os resultados são mostrados na Figura 2.

A primeira e mais importante revelação do gráfico é que, para todas as imagens de teste, a qualidade da segmentação foi consistentemente melhor quando o critério de heterogeneidade combinou um atributo morfológico com os atributos espectrais. O gráfico mostra também que para a imagem dos tanques (Fig. 1a) os melhores resultados foram alcançados quando se utilizaram os atributos compacidade e fator de forma circular, embora outros atributos tenham gerado resultados similares, com exceção dos atributos suavidade e anisometria, que produziram segmentações acentuadamente piores. 
Figura 2 - Discrepância $(D)$ das segmentações usando apenas atributos espectrais e com a introdução de um atributo morfológico.

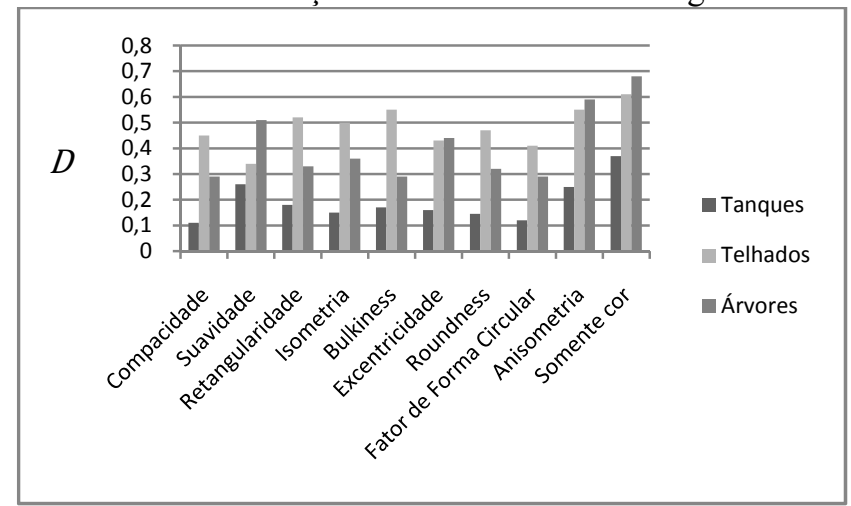

É interessante notar que os melhores resultados para a segmentação da imagem dos tanques foram alcançados exatamente com os atributos que capturam formas circulares, como é o caso das referências nestas imagens.

Já para a imagem dos telhados (Fig. 1b), foi com o atributo suavidade, associado aos atributos espectrais, que se alcançou o melhor desempenho. Com os outros atributos obtiveram-se valores de discrepância intermediários, tendo sido os atributos bulkiness e anisometria aqueles com que se produziram as piores segmentações.

Finalmente, para a imagem das árvores (Fig. 1c), o desempenho foi semelhante para a maioria dos atributos morfológicos, tendo sido o fator de forma circular aquele que produziu melhor resultado, seguido pela compacidade. Com os atributos suavidade e anisometria se obteve discrepância muito superior, ainda que menor do que a que se alcançou utilizando apenas atributos espectrais.

Tais resultados sugerem que a escolha do atributo morfológico a ser incorporado no critério que conduz o crescimento de regiões pode ter impacto significativo no resultado da segmentação.

Os resultados revelam ainda que, dependendo da classe de objetos a ser reconhecida, pode haver atributos mais adequados do que a suavidade ou a compacidade para descrever a forma dos segmentos. Nota-se que, quando os alvos que se busca delinear pela segmentação têm uma forma típica, como é o caso dos tanques (Fig. 1a) e das copas das árvores (Fig. 1c), convém considerar atributos morfológicos que alcançam seu valor mínimo para essa forma. Justifica-se, portanto, a extensão ao algoritmo de Baatz e Schäpe (2000) proposta neste estudo que propicia mais opções para a escolha do(s) atributo(s) morfológico(s) que compõe $(\mathrm{m})$ o critério de heterogeneidade.

A Figura 3 mostra para cada imagem o menor e o maior valor de discrepância trazido pela introdução de um atributo morfológico no critério de heterogeneidade 
do algoritmo de segmentação em relação à opção em que se consideraram apenas atributos espectrais.

Figura 3 - Comparação da discrepância $(D)$ as segmentações usando apenas atributos espectrais com as de menor e maior discrepância obtidas com a introdução de um atributo morfológico.

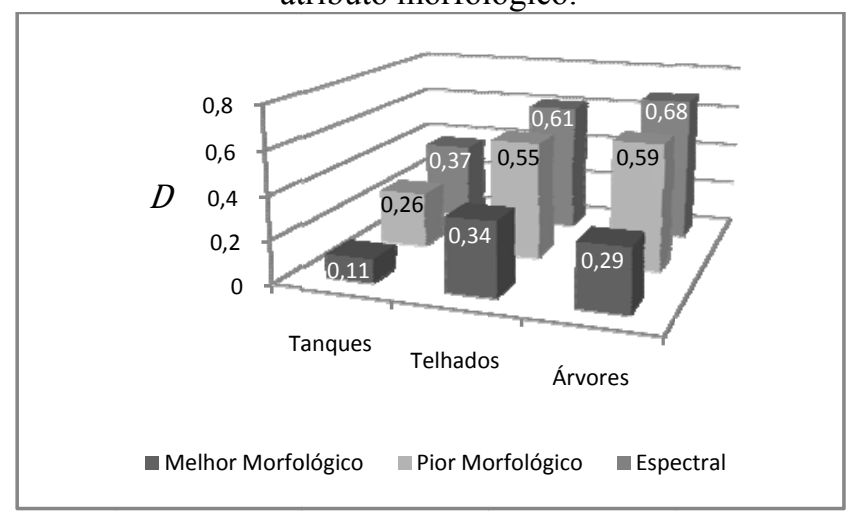

Figura 4 - Imagem dos tanques: segmentação somente espectral (a), segmentação com o atributo morfológico melhor avaliado (compacidade) (b) e pior avaliado (suavidade) (c).

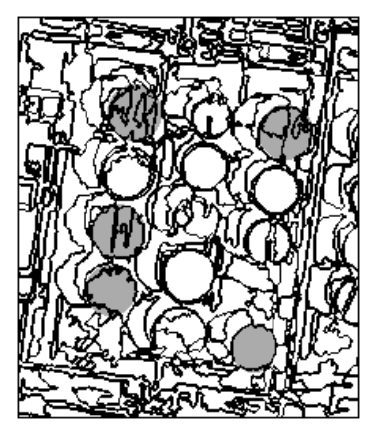

(a)

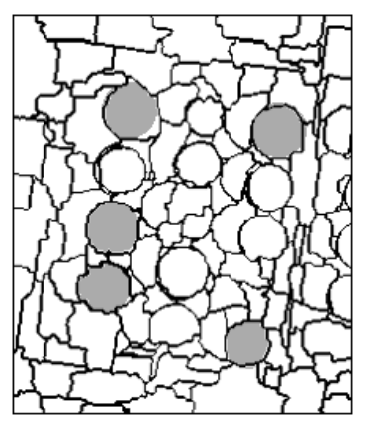

(b)

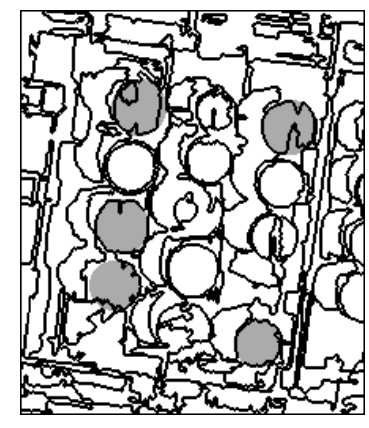

(c)

A relação entre a métrica definida pela Equação 14 e a percepção visual quanto à qualidade da segmentação está longe de ser evidente. É, portanto, importante verificar como se traduzem visualmente as diferenças nos índices de discrepância apresentados nas Figuras 2 e 3.

No caso da Figura 1a as segmentações têm como referência tanques com forma circular, mas espectralmente heterogêneos, o que dificultou a segmentação 
usando apenas atributos espectrais. A Figura 4a mostra que a segmentação somente espectral levou à supersegmentação e que as fronteiras dos segmentos acompanharam, em geral, as variações espectrais.

A introdução do atributo compacidade, que reduziu o índice de discrepância de 0,37 para 0,11, produziu o resultado mostrado na Figura 4b. Comparando-se com a Figura 4a, nota-se que os segmentos se ajustam bem melhor às referências, com exceção de pequenas discordâncias nas bordas dos segmentos. Com o atributo suavidade o índice chegou a $D=0,26$ (Fig. 4c). A análise visual dos resultados mostra um ganho de qualidade importante em relação à segmentação puramente espectral (Fig. 4a), mas ainda muito inferior ao apresentado na Figura 4b, quando se utilizou a compacidade.

Figura 5 - Imagem dos telhados: segmentação somente espectral (a), segmentação com o atributo morfológico melhor avaliado (suavidade) (b) e pior avaliado (anisometria) (c).

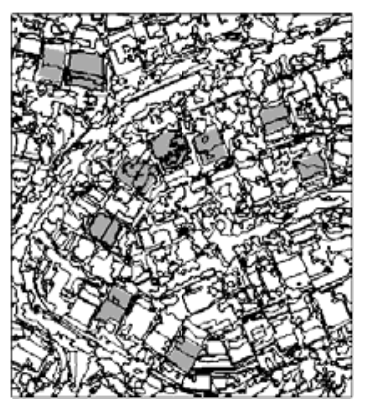

(a)

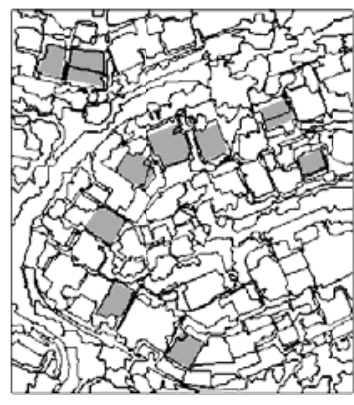

(b)

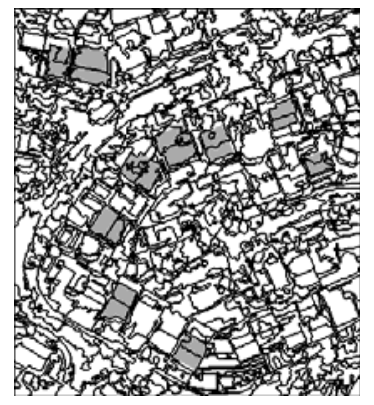

(c)

Para a imagem da Figura 1b (telhados), a textura, ainda que suave, e a variação de iluminação causada pelos ângulos de inclinação distintos das águas dos telhados dificultaram uma boa segmentação baseada apenas na cor.

A Figura 5a, que apresenta o resultado a partir apenas de atributos espectrais, evidencia essa dificuldade. Nota-se supersegmentação, tanto devido à cumeeira dos telhados em algumas referências, quanto à variação de iluminação e textura em outras. Novamente neste caso, o contorno dos objetos se apresenta irregular.

Com a introdução do atributo suavidade (Fig. 5b), o índice de discrepância passou de 0,61 para 0,34. Os contornos dos segmentos se mostram mais coerentes com as referências e mais suaves, ainda que algumas referências tenham permanecido divididas em dois segmentos na linha da cumeeira do telhado e alguns segmentos tenham englobado áreas adjacentes aos telhados com característica espectral semelhante. 
A segmentação obtida com o atributo anisometria (Fig. 5c) foi a que produziu a pior avaliação, mais exatamente $D=0,55$. Esta segmentação foi ligeiramente melhor do que a obtida somente com atributos espectrais, mas ainda apresentou supersegmentação e contornos irregulares.

Figura 6 - Imagem das árvores: segmentação somente espectral (a), segmentação com o atributo morfológico melhor avaliado (fator de forma circular) (b) e pior avaliado (anisometria) (c).

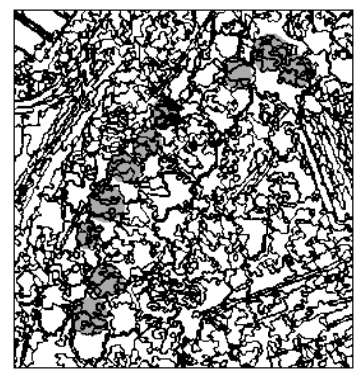

(a)

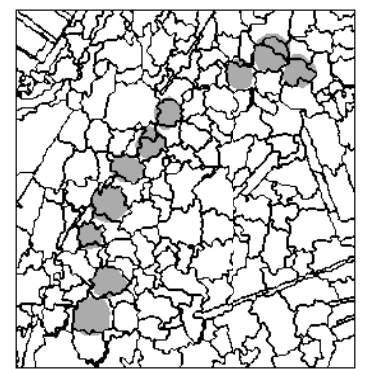

(b)

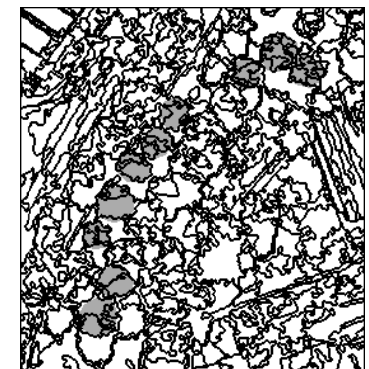

(c)

Para a imagem das árvores, a textura inerente da folhagem das copas e a variação de iluminação também tornaram difícil uma boa segmentação baseada apenas na cor.

A Figura 6a apresenta o resultado da segmentação puramente espectral. De maneira semelhante às imagens anteriores, nota-se uma acentuada supersegmentação e contornos de segmentos bastante irregulares e ramificados.

A segmentação produzida com a introdução do atributo fator de forma circular (Fig. 6b) produziu a melhor avaliação, reduzindo o índice de discrepância de 0,68 para 0,29 . Nesta figura, os contornos dos segmentos são bem menos irregulares e aderem melhor às referências fornecidas.

A segmentação obtida com o atributo anisometria (Fig. 6c) foi a pior, tendo atingido o valor $D=0,59$. Esta segmentação foi ligeiramente melhor do que a segmentação obtida somente com atributos espectrais, mas continuou apresentando supersegmentação e contornos irregulares.

Em suma, a análise visual dos resultados corrobora, portanto, a conclusão já aventada com base nos valores numéricos do índice de discrepância, ou seja, a de que a inclusão de atributos morfológicos na formulação da heterogeneidade pode melhorar significativamente a qualidade da segmentação. As segmentações com atributos de forma mostraram-se mais coerentes com as referências, mesmo para a imagem dos telhados em que o ganho foi menor. A inclusão de atributos morfológicos contribuiu em todos os casos para reduzir a supersegmentação. 
A análise visual confirma igualmente a conclusão já cogitada nesta seção de que a escolha do parâmetro morfológico que compõe a medida de heterogeneidade pode ser crítica para o resultado final da segmentação.

\subsection{Segmentação com base em dois atributos morfológicos e em atributos espectrais}

Na segunda série de experimentos foram testados critérios de heterogeneidade que incorporam os atributos espectrais, o melhor atributo morfológico identificado na série de experimentos anterior e um segundo atributo morfológico.

Três parâmetros foram estimados: parâmetro de escala $E$, peso da forma $w_{\text {forma }}$ e o peso $w_{s}$. Apenas o peso de um atributo foi otimizado, pois o peso do outro atributo foi definido como $1-w_{s}$. O método GPS foi usado novamente para encontrar o conjunto ótimo de valores destes parâmetros. Novamente, dez experimentos foram realizados para cada configuração e a melhor avaliação ( $D$ mínimo) foi considerada.

Figura 7 - Avaliações das segmentações obtidas com a introdução de um e dois atributos de forma para a imagem das árvores.

(*Atributo que obteve melhor avaliação nos experimentos anteriores).

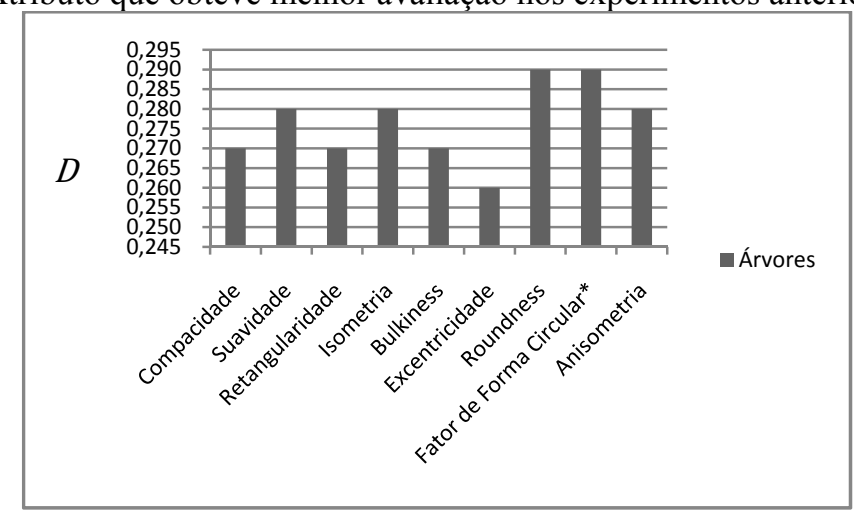

Para as imagens dos tanques (Fig. 1a) e dos telhados (Fig. 1c) não se observa nenhum ganho significativo na qualidade das segmentações decorrente da inclusão de um segundo atributo morfológico à medida de heterogeneidade. Nesses casos, o processo de otimização igualou a 0 (zero) o peso do segundo atributo, produzindo segmentações semelhantes às do experimento anterior. Entretanto, para as imagens das árvores, a introdução do segundo atributo morfológico levou a segmentações com melhores avaliações (Figura 7). Neste caso, a combinação do atributo fator de forma circular, que produziu a melhor avaliação nos experimentos anteriores, com os outros atributos levou a melhores segmentações em quase todos os casos, com exceção do atributo roundness, que não produziu ganho. A combinação que trouxe o 
maior benefício foi com o atributo excentricidade. O índice de discrepância passou de 0,29 quando se utilizou apenas fator de forma circular, para 0,26 quando a ele se agregou a excentricidade.

A Figura 8 mostra os resultados obtidos para a imagem das árvores apenas com atributos espectrais, combinados com um e com dois atributos morfológicos. Embora a introdução do segundo atributo morfológico não tenha produzido nenhum ganho para as outras duas imagens, uma avaliação visual dos resultados obtidos para esta imagem evidencia uma melhora decorrente do acréscimo do segundo atributo morfológico.

Figura 8 - Imagem das árvores: segmentação somente espectral (a), segmentação com o atributo fator de forma circular (b) e com os atributos fator de forma circular e excentricidade (c).

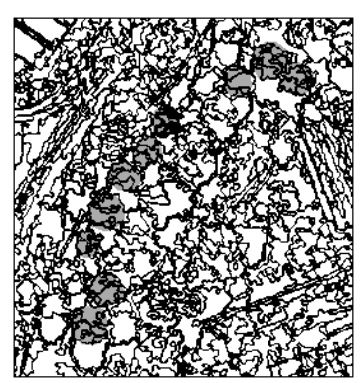

(a)

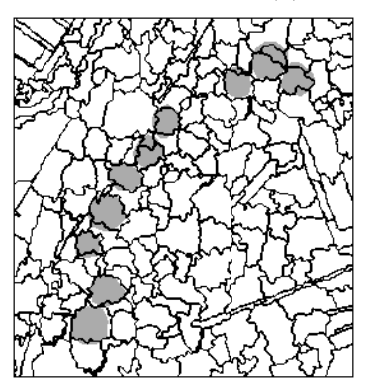

(b)

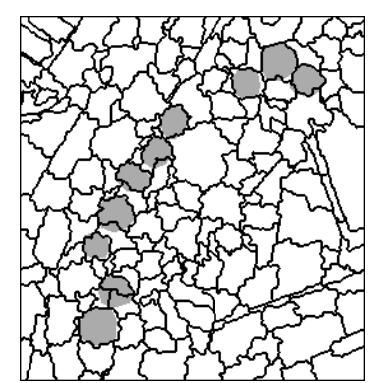

(c)

\section{CONCLUSÕES}

Neste estudo, avaliou-se a melhoria da qualidade da segmentação decorrente de se incorporarem atributos morfológicos, além dos espectrais, no critério de heterogeneidade que governa o crescimento de regiões.

Para tanto, foi proposta e implementada uma extensão do segmentador multiresolução descrito em (BAATZ; SCHÄPE, 2000), que permite a inclusão de diferentes atributos morfológicos na formulação do critério de heterogeneidade.

Em todos os experimentos realizados a qualidade da segmentação melhorou quando o critério de heterogeneidade que rege o crescimento de regiões leva em conta atributos morfológicos, além dos espectrais. $\mathrm{O}$ estudo mostrou ainda que o ganho de qualidade pode ser significativo dependendo da forma dos alvos a serem delineados pela segmentação e do atributo morfológico utilizado.

Ao mesmo tempo, os experimentos indicaram um ganho de qualidade que não pode ser desprezado quando se acrescenta um segundo atributo morfológico na composição do critério de heterogeneidade do segmentador. 
Os resultados sugerem ainda que atributos que alcançam seu valor mínimo para a forma típica dos alvos a serem delineados têm maior potencial de melhorarem o resultado da segmentação.

Há que se considerar, contudo, que em aplicações reais as classes de objeto a serem reconhecidas nem sempre apresentam uma forma típica. Mais raro ainda é o caso em que todas as classes de objeto presentes na imagem apresentam a mesma forma típica. Consequentemente, em geral o conjunto ótimo de parâmetros de segmentação será distinto para cada classe de objetos. Este fato contribui para que, na maioria das aplicações práticas, o objetivo de encontrar uma segmentação adequada para todos os tipos de objetos presentes na imagem se torne virtualmente inatingível, mesmo que o critério de heterogeneidade incorpore atributos morfológicos, como se considerou neste estudo. Por outro lado, a utilização simultânea de segmentações diferentes otimizadas para cada classe de objetos, traz consigo problemas adicionais, cuja investigação excede o escopo deste trabalho.

É importante ressaltar que o presente estudo se limitou à qualidade da segmentação. Avaliar como as melhorias na segmentação aqui detectadas impactam a acurácia final da classificação é uma questão a ser considerada na continuação desta investigação.

Cabe por fim registrar que o programa de segmentação implementado permite a introdução de qualquer novo atributo morfológico na composição do critério de heterogeneidade, viabilizando eventuais estudos futuros sobre outros atributos, com outras imagens e classes de objetos-alvo. O programa de segmentação e seu códigofonte podem ser baixados neste endereço: http://www.lvc.ele.pucrio.br/wp/?p=1076.

\section{AGRADECIMENTOS:}

Os autores agradecem o apoio do Conselho Nacional de Desenvolvimento Científico e Tecnológico (CNPq) e do programa FP7 no âmbito do projeto TOLOMEO para a realização deste trabalho.

\section{REFERÊNCIAS BIBLIOGRÁFICAS}

ALVES, C. D.; PEREIRA, M. N.; FLORENZANO, T. G.; SOUZA, I. M. Análise Orientada a Objeto no Mapeamento de Áreas Urbanas com Imagens LANDSAT. Boletim de Ciências Geodésicas, Curitiba, v. 15, n. 1, p. 120-141, jan./mar. 2009.

BAATZ, M.; SCHÄPE, A. Multiresolution Segmentation: an optimization approach for high quality multi-scale image segmentation. Journal of Photogrammetry and Remote Sensing, Heidelberg, v. 58, n. 3-4, p. 12-23, 2000.

BAUM, R. P.; KULKARNI, H. R.; CARRERAS, C. Peptides and receptors in image-guided therapy: theranostics for neuroendocrine neoplasms. Seminars in Nuclear Medicine, v. 42, n. 3, p. 190-207, mai. 2012. 
BEAUCHEMIN, M.; THOMSON, K. P. B. The evaluation of segmentation results and the overlapping area matrix. International Journal of Remote Sensing, v. 18, n. 18, p. 3895-3899, 1997.

BLASCHKE, T.; STROBL, J. What is wrong with pixels? Some recent developments interfacing remote sensing and GIS. Image Rochester NY, v. 6, n. 6, p. 12-17, 2001.

BLASCHKE, T.; LANG, S.; HAY, G. (Eds.). Object-based Image Analysis: Spatial Concepts for Knowledge-Driven Remote Sensing Applications. Springer, 2008, p. 3-28. (Lecture Notes in Geoinformation and Cartography)

BRODSKÝ, L.; BORU゚VKA, L. Object-oriented Fuzzy Analysis of Remote Sensing Data for Bare Soil Brightness Mapping. Soil \& Water Research, v. 1, n. 3, p. 79-84, 2006.

CÂMARA, G.; SOUZA, R. C. M.; FrEITAS, U. M.; GARRIDO, J.; II, F. M. SPRING: Integrating remote sensing and GIS by object-oriented data modelling. Computers \& Graphics, v. 20, n. 3, p. 395-403, mai./jun. 1996.

CHABRIER, S., EMILE, B.; ROSENBERGER, C.; LAURENT, H. Unsupervised performance evaluation of image segmentation. EURASIP Journal on Applied Signal Processing, v. 2006, p. 1-12, mar. 2006.

ClintON, N., HOLT, A., SCARBOROUGH, J., YAN, L., GONG, P. Accuracy assessment measures for object-based image segmentation goodness. Photogrammetric Engineering and Remote Sensing v. 76, n. 3, p. 289-299, 2010.

COMANICIU, D.; MEER, P. Mean Shift: A Robust Approach Toward Feature Space Analysis. IEE Transactions on Pattern Analysis and Machine Intelligence, v. 24, n. 5, p. 603-619, mai. 2002.

DENNIS, J. E.; TORCZON, V. Direct Search Methods on Parallel Machines. SIAM Journal on Optimization, v. 1, p. 448-474, 1991.

FEITOSA, R. Q.; COSTA, G. A.; CAZES, T. B.; FEIJÓ, B. A Genetic Approach for the Automatic Adaptation of Segmentation Parameters. In: BRIDGING REMOTE SENSING AND GIS. 1st INTERNATIONAL CONFERENCE ON OBJECT-BASED IMAGE ANALYSIS (OBIA 2006), 2006, Salzburg. ISPRS Archives - Volume XXXVI-4/C42. Salzburg, 2006. 6 p. 1 CD. ISSN: 16821777

FELZENSZWALB, P. F.; HUTTENLOCHER, D. P. Efficient Graph-Based Image Segmentation. International Journal of Computer Vision, v. 59, n. 2, p. $167-$ $181,2004$.

FERREIRA, R. S. Uma Abordagem Multiescalar, Multicritério para a Segmentação de Imagens. 2011. 113 p. Dissertação (Mestrado em Engenharia Elétrica) - Departamento de Engenharia Elétrica, Pontifícia Universidade Católica do Rio de Janeiro, Rio de Janeiro. 2011.

FORCADEL, N.; GUYADER, C. L.; GOUT, C. Generalized fast marching method: applications to image segmentation. Numerical Algorithms, v. 48 n. 1-3, p. 189-211, jul. 2008.

Bol. Ciênc. Geod., sec. Artigos, Curitiba, v. 19, nº 3, p.452-471, jul-set, 2013. 
GOMES, O. F. M.; PACIORNIK, S. Automatic Classification of Graphite in Cast Iron. Microscopy and Microanalysis, v. 11, n. 4, p. 363-371, ago. 2005.

HALCON. Features [HALCON Reference Manual / Version 9.0.4]. Disponível em: $<$ http://www.halcon.de/halcon/download/documentation/reference9.0/hdevelop/toc_regions_features.html> Acesso em: 31 de ago. 2012.

HARALICK, R. M.; SHAPIRO, L. G. Image Segmentation Techniques. Computer Vision, Graphics, and Image Processing, v. 29, n. 1, p. 100-132, jan. 1985.

HOOKE, R.; JEEVES, T. A. "Direct Search" Solution of Numerical and Statistical Problems. Journal of the ACM, v. 8, n. 2, p. 212-229, abr. 1961.

LIU, Y.; BIAN, L.; MENG, Y.; WANG, H.; ZHANG, S.; YANG, Y.; SHAO, X.; WANG, B. Discrepancy measures for selecting optimal combination of parameter values in object-based image analysis. ISPRS Journal of Photogrammetry and Remote Sensing, v. 68, p. 144-156, mar. 2012.

MARPU, P. R.; NEUBERT, M.; HEROLD, H.; NIEMEYER, I. Enhanced evaluation of image segmentation results. Journal of Spatial Science, v. 55, n. 1, p. 55-68, 2010.

MEINEL, G.; NEUBERT, M. A comparison of segmentation programs for high resolution remote sensing data. In: XXth ISPRS CONGRESS, 2004, Istanbul. ISPRS Archives - Volume XXXV Part B4. Istanbul, 2004. p. 1097-1102. ISSN: 1682-1750

NEUBERT, M.; HEROLD, H; MEINEL, G. Evaluation of Remote Sensing Image Segmentation Quality - Further Results and Concepts. In: BRIDGING REMOTE SENSING AND GIS. 1st INTERNATIONAL CONFERENCE ON OBJECT-BASED IMAGE ANALYSIS (OBIA 2006), 2006, Salzburg. ISPRS Archives - Volume XXXVI-4/C42. Salzburg, 2006. 6 p. 1 CD. ISSN: 16821777

NEUBERT, M.; HEROLD, H.; MEINEL, G. Assessment of Remote Sensing Image Segmentation Quality. In: GEOBIA 2008 - PIXELS, OBJECTS, INTELLIGENCE. GEOBRAPHIC OBJECT BASED IMAGE ANALYSIS FOR THE 21ST CENTURY, 2008, Calgary. ISPRS Archives - Volume XXXVIII-4/C1. Calgary, 2008. 5 p. ISSN: 1682-1777

RUSS, J. C. The Image Processing Handbook. 3. ed. Boca Raton: CRC Press, 1998. $885 \mathrm{p}$.

TILTON, J. C. Image Segmentation by Iterative Parallel Region Growing and Splitting. In: GEOSCIENCE AND REMOTE SENSING SYMPOSIUM, 1989, Vancouver. Proceedings... Vancouver, 1989. p. 2420-2423.

TORCZON, V. On the Convergence of Pattern Search Algorithms. SIAM Journal on Optimization, v. 7, n. 1, p. 1-25, fev. 1997.

VESE, L. A.; CHAN, T. F. A Multiphase Level Set Framework for Image Segmentation Using the Mumford and Shah Model. International Journal of Computer Vision, v. 50, p. 271-293, 2002.

(Recebido em outubro de 2012. Aceito em julho de 2013). 